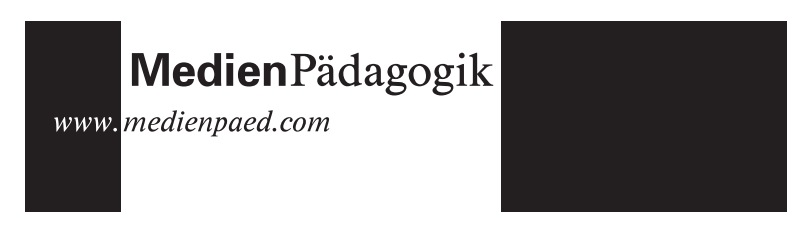

Rainer Winter

6.2 .2004

\section{Cultural Studies und kritische Pädagogik}

\section{Einleitung}

Die Geschichte von Cultural Studies zeigt, dass dieses intellektuelle und politische Projekt von Anfang an eng verbunden mit Fragen der Erziehung und der Pädagogik war. Denn es entstand im vitalen und intellektuell vielseitigen Milieu der Erwachsenenbildung in den 50er Jahren in Grossbritannien (vgl. Grossberg 1997, Steele 1997). Im produktiven Austausch mit reifen Studenten aus der Arbeiterklasse entwickelten Edward P. Thompson, Raymond Williams und Richard Hoggart ihre kreativen Ideen zur Kulturanalyse und schrieben die grundlegenden Werke dieser neuen transdisziplinären Forschungsrichtung, die - wie die Erwachsenenbildungdie Spezialisierung des Wissens überwinden und dessen mögliche praktische Relevanz hervorheben möchte.

Leitend war dabei ein umfassender Kulturbegriff, der besonders in den Arbeiten von Williams theoretische Kontur gewann, so z. B. in Culture and Society 1780-1950 (1958) und in The Long Revolution (1961). Er arbeitete die Vielschichtigkeit und Komplexität von Kultur heraus, die nicht nur die Hochkultur umfasst, sondern auch die Populärkultur. Indem er zudem Kultur in einem anthropologischen Sinne als «ganze Lebensweise» [a whole way of life] begriff, lenkte er die Aufmerksamkeit auf ihre Verbindungen und Vernetzungen mit anderen gesellschaftlichen Praktiken. Wie Thompson hob er die besondere Rolle der «Erfahrung» in der Kulturanalyse hervor, denn sie ermöglicht ein Wissen über und einen Zugang zu kulturellen und gesellschaftlichen Zusammenhängen, die durch abstrakte Theorien nicht erworben werden können.

Im Kontext der Erwachsenenbildung, wie in der «workers' education», waren die Rollen von Professoren und Studenten nicht so hierarchisch definiert wie an der Universität. Diese nicht traditionellen Studenten, denen der Zugang zur «higher education» verwehrt war, akzeptierten nicht zwangsläufig die Autorität des Professors, bezogen das Gelernte auf ihr eigenes Leben und stellten im Unterricht Fragen, die praktische Relevanz für ihre eigene Erfahrung hatten (Williams 1989: 152) und die Grenzen von akademischen Disziplinen nicht akzeptierten. Diese «radikale Herausforderung» (Grossberg 2000: 12) führte nicht nur dazu, dass neben der Literatur u. a. die Presse, das Radio und der Film zum Thema wurden, sondern dass es auch möglich wurde, die Studenten dazu anzuleiten, ihr eigenes Leben im Kontext der gesellschaftlichen Verhältnisse zu begreifen, und in einem zweiten Schritt Möglichkeiten aufzuzeigen, wie es verändert werden kann, um mehr soziale Gerechtigkeit und Gleichheit herzustellen. Es waren diese zur Universität alternativen Institutionen, die in Grossbritannien einen Raum für Cultural Studies eröffneten.

Hierbei ist Kultur, die in neueren Studien als ein «network of embedded practices and representations (texts, images, talk, codes of behavior, and the narrative structures organizing these)»(Frow/Morris 2000: 316) bestimmt wird, der Ort, an dem Machtverhältnisse stabilisiert, aber auch in Frage gestellt und verändert werden können. Cultural Studies analysieren also nicht nur, sondern sie haben auch einen interventionistischen Charakter (Grossberg/Nelson/Treichler 1992). An die Stelle der Arbeiterklasse sind seit den 60er Jahren neue soziale Bewegungen, marginalisierte Minoritäten und unterdrückte Gruppen getreten, deren «agency» gesteigert werden soll, indem sie lernen, ihre konkrete Lebenssituation gesellschaftlich zu kontextualisieren und Möglichkeiten der Veränderung zu erkennen und zu ergreifen.

Der Zusammenhang von Cultural Studies und einer kritischen Pädagogik soll im folgenden in vier Schritten analysiert werden. Zunächst möchte ich kurz auf die eher randständige Bedeutung der Pädagogik am Centre for Contemporary Cultural Studies in den 60er und 70er Jahren eingehen (1). Anschliessend werde ich die (amerikanische) Weiterentwicklung des dort entwickelten «ethnography of reading»-Ansatzes untersuchen und seine pädagogischen Implikationen herausarbeiten (2). Dann werde ich die Verbindungen von Cultural Studies und der in den USA beheimateten kritischen Pädagogik erörtern. Diese radikale Theorie der Erziehung wird von einigen ihrer Vertreter in neueren Arbeiten als die «practical politics of cultural studies» bestimmt (3). Eine kurze Zusammenfassung beendet den Beitrag (4) 
1. Die Arbeit des CCCS und ihre pädagogischen Implikationen

Am Birmingham Centre for Contemporary Cultural Studies, das zunächst von Richard Hoggart und später von Stuart Hall geleitet wurde, der auch aus der Erwachsenenbildung kam und zur New Left gehörte, wurden «media studies», die Analyse von Film und Fernsehen, der Presse etc. zu wichtigen Themen. Fragen der Pädagogik wurden explizit allerdings nur am Rande behandelt, auch wenn das Centre für seine Jugendstudien weltberühmt wurde. So stellt Lawrence Grossberg fest: «There is in fact very little explicit work published in either the 'Occasional Papers' or the more formal Working Papers published by the Centre for Contemporary Cultural Studies during the 1970s» (Grossberg 1997: 376). Trotzdem lassen die beiden wesentlichen Forschungsrichtungen, die Jugendstudien mit ihrem Modell von Inkorporation und Widerstand auf der einen Seite, die Medienforschungen mit ihrer ideologiekritischen Analyse medialer Texte auf der anderen Seite, Merkmale erkennen, die für eine kritische Pädagogik von Relevanz sind.

So wird sowohl in bezug auf die Jugendlichen aus der Arbeiterklasse, als auch in bezug auf Fernsehzuschauer gezeigt, dass sie keine «cultural dopes» sind, sondern im Umgang mit vorgegebenen Produkten bzw. kulturellen Waren ihre eigenen Kulturen schaffen. Zweifellos liegt in Birmingham der Schwerpunkt auf der «agency», die immer schon zumindest rudimentär vorhanden ist, aber auch durch soziale Bedingungen eingeschränkt wird. Die Populärkultur wird im Anschluss an Gramscis Hegemoniekonzept zur «zone of contestation». «Popular culture is one of the sites where this struggle for and against a culture of the powerful is engaged: it is also the stake to be won or lost in that struggle. It is the arena of consent and resistance» (Hall 1999). Die interdisziplinären Untersuchungen von Cultural Studies zielen darauf, die «agency» zu erweitern, indem sie z. B. zeigen, wie Nachrichten im Fernsehen ideologisch strukturiert sind und wie vor dem Hintergrund der eigenen Interessen kritisch mit ihnen umgegangen werden kann.

Da die Forschungen ihren Ausgangspunkt in konkreten Fragestellungen von praktischer Relevanz haben, fällt es nicht schwer, die Verbindungen zu den Lebenswelten der Untersuchten herzustellen. Dabei beschränken Cultural Studies sich nicht auf die Analyse kultureller Objekte oder Institutionen, sondern untersuchen, wie Menschen in unterschiedlichen Kontexten Kultur schaffen und erfahren, wobei sie deren produktive und transformative Kraft ins Zentrum rücken. «Cultural studies assumes a reality that is constantly reworked by and only made available through cultural practices» (Grossberg 1997: 379).

Stuart Hall hat gezeigt, dass sich auf theoretischer Ebene das Projekt von Cultural Studies zwischen den Paradigmen von Kulturalismus und Strukturalismus entfaltet, was sich sowohl an den Jugend- als auch an den Medienstudien sehr gut zeigen lässt (Winter 2001). Sie machen nämlich deutlich, dass Strukturen nicht ahistorisch und stabil sind, sondern sie sind immer «structures-in-use», wobei die «uses» nicht im voraus bestimmt werden können (Frow/Morris 2000: 326). Zur wissenschaftlichen Untersuchung von aktuellen Fragestellungen, die sich im britischen Kontext ergaben, griffen die Vertreter des CCCS eklektisch und innovativ auf unterschiedliche, dafür geeignete Theorien und Methoden zurück, so dass der Forschungsprozess, wie es bei qualitativer Forschung oft der Fall ist, zu einer Bricolage wurde (Denzin 1994).

Das auf soziale Veränderung zielende interventionistische Motiv von Cultural Studies wird in den rückblickend von Stuart Hall geäusserten Worten deutlich: «Our questions about culture (...) were concerned with the changing ways of life of societies and groups and the networks of meanings that individuals and groups use to make sense of and to communicate with one another (...) cultural studies insists on the necessity to address the central, urgent, and disturbing questions of a society and a culture in the most rigorous intellectual way we have available» (Hall 1996: 336f.). Zum einen wird hier klar, warum das Birmingham Projekt eine kritische Pädagogik impliziert, auch wenn sie nicht ausbuchstabiert ist, zum anderen wird auch verständlich, warum Vertreter der in den USA beheimateten Varianten kritischer Pädagogik, die in Auseinandersetzung mit der Tradition des westlichen Marxismus, so mit der Frankfurter Schule, entstanden sind, seit den 80er Jahren an die Cultural Studies anknüpfen. Bevor wir uns diesen Ansätzen zuwenden, soll im folgenden an einem Beispiel die Weiterentwicklung der in Birmingham entwickelten «audience ethnography» in den USA näher betrachtet werden, da auch sie für unsere Fragestellung von besonderer Relevanz ist.

\section{Audience Ethnography: Polysemie und plurale Gebrauchsweisen}

Stuart Halls «Encoding-Decoding» Modell und die an ihn anschliessenden Studien von David Morley schufen die Grundlage für einen äusserst fruchtbaren und innovativen Ansatz in der Medienforschung: die «audience ethnography» (vgl. Hay/Grossberg/Wartella 1996). Wenig diskutiert wurde 
bisher dessen pädagogisches Potential. Es war vor allem John Fiske (1987, 1989), der in seinen synthetisierenden Arbeiten Ende der 80er Jahre, ausgehend von einer dekonstruktiven Analyse von Fernsehtexten deren polysemen Charakter herausarbeitete, um das heterogene Potential pluraler Aneignungsformen sichtbar $\mathrm{zu}$ machen, das je nach sozialer und historischer Situation der Zuschauer von diesen unterschiedlich realisiert werden kann. Die Rezeption und die Aneignung von Texten wird in seiner Lesart zu einer kontextuell verankerten gesellschaftlichen Praxis, in der die Texte als Objekte nicht vorgegeben sind, sondern erst auf der Basis sozialer Erfahrungen produziert werden. Dabei knüpft Fiske zum einen an die Arbeiten aus Birmingham an, zum anderen an Michel Foucaults Unterscheidung zwischen Macht und Widerstand und an de Michel de Certeaus Analyse kreativer Alltagspraktiken. ,Widerstand' kann in spezifischen historischen Situationen im Verhältnis von diskursiven Strukturen, kultureller Praxis und subjektiven Erfahrungen entstehen. Im eigensinnigen Gebrauch der «Ressourcen», die das (kapitalistische) System in Form von medialen Texten und anderen Konsumobjekten zur Verfügung stellt, versuchen die alltäglichen Akteure ihre Lebensbedingungen selbst zu definieren und ihre Interessen auszudrücken.

Vor allem in seinen späteren Analysen widmet Fiske (1993; 1994) sich spezifischen Raum-Zeit-Momenten des Mediengebrauchs und bestimmt die Einzigartigkeit und Signifikanz kultureller Praktiken, die an einem besonderen Ort zu einer besonderen Zeit realisiert werden. Damit reagierte er auf Kritiken, die ihm vorwarfen, er würde davon ausgehen, jeder Konsum populärer Medien wäre potentiell subversiv. Sinnvoller scheint es zu sein, Widerständigkeit als mögliche Option populärer Texte $\mathrm{zu}$ bestimmen, wobei geklärt werden muss, ob die subversive Artikulation von Bedeutungen auf den spezifischen Kontext der Medienrezeption beschränkt bleibt oder auch in anderen Bereichen des Alltags seine Wirkung entfaltet. Allerdings müssen die mobilisierten Gefühle und ausgehandelten Bedeutungen nicht zwangsläufig im Sinne von «empowerment» organisiert sein. Douglas Kellner (1995: 38f.) hebt in seiner Kritik hervor, dass zwischen verschiedenen Formen des Widerstandes unterschieden werden sollte. «Resistance and pleasure cannot therefore be valorized per se as progressive elements of the appropriation of cultural texts, rather one needs to describe the specific conditions that give rise to the resistance or pleasure at stake and their specific effects» (Kellner 1995: 39).

Ausserdem weist Grossberg darauf hin, dass untersucht werden sollte, wie das tägliche Leben mit der Politik der Gesellschaftsformation im ganzen artikuliert ist. «By valorizing daily life as intrinsically disruptive and playful, it constantly discovers moments of resistance, whether or not they have tangible effects. By equating structure and power, it creates the illusion that one can escape them [...] By equating resistance with the deconstruction of any structure, it often unconsciously reproduces those positions of power which, at any moment, for whatever reasons, have escaped the deconstructive onslaught»(Grossberg 1992: 94). Solche vereinfachenden Gleichsetzungen, die Anlass zu polemischen Kritiken von Gegnern der Cultural Studies geben, haben zur Folge, dass die Analyse des Verhältnisses zwischen den Formen des Vergnügens sowie der Bedeutungsproduktion, mit denen bis zu einem bestimmten Grad eine Kontrolle über die eigenen Lebensbedingungen ausgeübt und die Differenzen zu anderen behauptet werden können, und den Strukturen der Gesellschaftsformation oft nich erfolgt. Ausserdem reduziert sich die Analyseperspektive leicht darauf, Praktiken primär unter den Aspekten von Selbstermächtigung und Widerstand zu begreifen. Dagegen stellt Grossberg fest, dass die lustvolle Aneignung von Texten dominante Vorzugsbedeutungen nicht zum Verschwinden bringen muss. Semiotischer Widerstand muss nicht in politische Praxis münden. «And struggle is not always resistance, which requires a specific antagonism. And resistance is not always opposition, which involves an active and explicit challenge to some structure of power» (Grossberg 1992: 95f.)

Trotz der zum Teil berechtigten Kritik an der «audience ethnography» führt diese innovative Forschungsrichtung aber anschaulich vor, dass sich die für den Alltag relevante Bedeutung von Texten in deren sozialen Gebrauch realisiert. Freilich, ist die Rezeption und Aneignung in der postmodernen Medienwelt sowie die Subjektivität der Konsumenten unterschiedlichen bestimmenden Einflüssen ausgesetzt. Dabei gilt das pädagogische Interesse von Cultural Studies aber primär den Bedeutungen und Vergnügen, die Personen und Gruppen helfen können, ihre Interessen zu artikulieren, Freiräume zu entfalten, Fluchtlinien zu finden und ihre Handlungsmächtigkeit zu erweitern. Auf diese Weise sind Texte in die Zirkulation von Bedeutungen und affektiven Energien innerhalb einer Kultur eingebunden. Das politische Ziel von Cultural Studies ist gerade die Herstellung von Zusammenhängen zwischen den einzelnen Momenten der Selbstermächtigung und den umfassenderen kulturellen und gesellschaftlichen Prozessen. Hierzu ist jedoch auch eine Kritik an den bestehenden 
Herrschaftsverhältnissen erforderlich und eine Analyse der Möglichkeiten zur sozialen Transformation. «An insurgent cultural studies only positively interprets struggle and resistance against oppression, against structural inequality, grounding its analyses in antagonisms between structural inequalities and oppression. An insurgent cultural studies enters into dialogue with members of oppressed groups in struggle and expands cultural studies to include voices usually excluded in more academic forms of cultural studies, thus striving for a more inclusive and political project» (Kellner 1995: 96). Vor allem der in den USA entwickelte Ansatz der kritischen Pädagogik beschäftigt sich explizit mit diesem Ziel und der Herstellung einer radikalen Demokratie.

\section{Kritische Pädagogik als Cultural Studies}

Ausgangspunkt der kritischen Pädagogik in den USA zu Beginn der 80er Jahre waren die von Bourdieu/Passeron und anderen durchgeführten Untersuchungen des Bildungswesens, die zeigen, dass es zur sozialen Reproduktion und zur Aufrechterhaltung bestehender Verhältnisse beiträgt. Die Vertreter dieser ideologiekritischen Richtung (Apple 1979, 1982; Aronowitz/ Giroux 1985) beschränkten sich jedoch nicht darauf, soziale Reproduktion als strukturellen Effekt zu analysieren. In Auseinandersetzung mit den theoretischen und empirischen Arbeiten aus Birmingham, insbesondere mit der Rezeption von Gramscis Hegemonie-Konzept und den Jugendsubkulturstudien, wurden Schulen vielmehr als hegemoniale Orte von Praktiken sowie Ritualen und Ideologien als gelebte Erfahrung und Praxis analysiert, insbesondere in der grundlegenden Arbeit Schooling as a Ritual Performace (1986) von Peter McLaren. Wie bereits Paul Willis (1977) zeigte, ist die Erfahrung der sozialen Welt nicht von externen Determinanten ableitbar, sondern sie ist widersprüchlich, uneinheitlich und veränderbar. Die Kultur ist der Bereich, in dem die Strukturen erfahren und gelebt, reproduziert, jedoch auch transformiert werden. Gerade hier setzt die kritische Pädagogik an, welche die kritische Handlungsfähigkeit von Schülern und Schülerinnen entwickeln und stützen möchte, um Strategien des kulturellen und politischen Widerstandes zu entwickeln. Dabei gibt es ganz unterschiedliche Positionen (Carlson/Apple 1998). Im folgenden werden wir primär auf die eingehen, die dem Projekt von Cultural Studies am nächsten stehen und es explizit mit der kritischen Pädagogik verbinden. So vermittelt Henry Giroux, einer der führenden Vertreter dieser Synthese, bereits in einer frühen Arbeit zwischen Kulturalismus und Strukturalismus bei seinem Bemühen, die Ideologiekritik in die Unterrichtspraxis einzubringen. Hierzu sollen die Schüler und Schülerinnen in der Klasse über ihre sozialen Erfahrungen reflektieren. «It is crucial for them to be able to understand how their own experiences are reinforced, contradicted, and suppressed as a result of the ideologies mediated in the material and intellectual practices that characterize daily classroom life» (Giroux 1983: 150). In einem Prozess dialogischen Lernens (Freire 1973) sollen sie zunächst ihr Selbst dekonstruieren, indem sie es im Kontext der gesellschaftlichen Verhältnisse begreifen. Dies ist die Voraussetzung dafür, zum potentiellen Akteur im Geschichtsprozess zu werden und durch Kampf und Kritik die bestehenden Verhältnisse zu ändern. Giroux' Pädagogik des Widerstandes ist auf die Transformation der Gesellschaft ausgerichtet und so mit Hoffnung, Transzendenz und Utopie verbunden.

Eine intensive Beschäftigung mit poststrukturalistischen, postmodernen und postkolonialen Ansätzen innerhalb der Cultural Studies führte Giroux, wie auch Peter McLaren (1995), allmählich zu einer Transformation seines Ansatzes (Giroux et al. 1984, Giroux 1992, 1993, 1994). Er vertritt heute eine explizit an Cultural Studies orientierte kritische Pädagogik, die eine Politik der Differenz mit einer Forderung nach einer radikalen Demokratisierung der Gesellschaft verbindet (Kellner 2001). Zum einen hebt er die wichtige Bedeutung von Cultural Studies für das Verständnis von Erziehung, Kultur und Politik hervor. Dabei ist sein Bemühen, die Pädagogik zu einem wesentlichen Bestandteil von Cultural Studies zu machen. Er plädiert für eine «politics of the performative»(...) a work in progress informed by a cultural politics that translates knowledge back into practice, places theory in the political space of the performative, and invigorates the pedagogical as a practice through which collective struggles can be waged to revive and maintain the fabric of democratic institutions» (Giroux 2000: 135). Andererseits kritisiert Giroux die Selbstgenügsamkeit der «textualist readings» und der «audience studies», die sich auf die Analyse des lustvollen, subversiven Gebrauchs von Medien beschränken. So hebt er z. B. in einer Analyse des Disney-Imperiums hervor, dass viele Disney-Texte wohl zu oppositionellen Lesarten einladen, dass diese jedoch dessen Macht, «to monopolize the media and saturate everyday life with its own ideologies» (Giroux 1999: 7), nicht zum Verschwinden bringen. Daher können die «audience studies» von der kritischen Pädagogik lernen, dass kreative und subversive Lesarten während der Rezeption und Aneignung nicht genügen, um mehr Demokratie zu verwirklichen. 
Die kritische Pädagogik macht vor allem die Aushandlung [negotiation] und die Produktion von Bedeutungen zwischen Lehrern und Schülern zu ihrem Thema, die sie im Kontext von diskursiven Praktiken und Macht/ Wissen Beziehungen kritisch analysiert. «By conceptualizing radical pedagogy as a form of cultural politics, we are underscoring the idea that school culture is not neutral, but ideological. It consists of stipulated social practices and diffuse configurations of power, as well as historically mediated ideas and world-views that often work to sustain the interests of dominant groups» (Giroux/McLaren 1995: 36).

Im Zeitalter des Neoliberalismus und der zunehmenden Privatisierung öffentlicher Räume ist, wie Giroux (2001) und auch Peter McLaren (1995, 1997) zeigen, die Entwicklung einer Ethik erforderlich, die das Verhältnis von Macht, subordinierten Subjektpositionen und gesellschaftlichen Praktiken reflektiert. «Critical pedagogy commits itself to forms of learning and action that are undertaken in solidarity with subordinated and marginalized groups» (Giroux/McLaren 1995: 32). Ausgehend von den aktuellen gesellschaftlichen Konflikten soll der ethische Diskurs nicht nur (ethnische) Differenzen anerkennen, sondern auch zeigen, wie Gerechtigkeit verwirklichbar ist. Weiterhin sollen sich die Lernenden mit der Vielfalt von Erzählungen und Traditionen, die die heutigen multikulturellen Gesellschaften kennzeichnen, auseinandersetzen und die Geschichte sowie ihre eigene Subjektivität als Ort von gesellschaftlichen Kämpfen begreifen. So sollen Studenten verstehen lernen, wie sich «conflictual social relations» ihrem Habitus eingeschrieben haben. «The task of critical pedagogy is to increase our self-consciousness, to strip away distortion, to discover modes of subjectivity which cohere in the capitalist body/subject and to assist the subject in its historical remaking» (McLaren 1995: 74). Auf diese Weise soll die «agency» der Studenten erweitert werden. Zum einen ist die kritische Pädagogik eine kulturelle Praxis, zum anderen eine Form des gesellschaftlichen Gedächtnisses. Dies wird besonders in dem Projekt der «postmodern counternarratives» ersichtlich (Giroux et al. 1996), in dem Cultural Studies selbst als eine «counternarrative» bestimmt werden, die die technokratische und marktorientierte Rationalität im Lehren und Lernen zugunsten einer demokratischen Aneignung von Wissen und kulturellen Texten zurückweist, was in eine scharfe Kritik an der «corporate university» mündet (vgl. Giroux/ Myrsiades 2001)

Unter verschiedenen politischen Blickwinkeln leitet die kritische Päda- gogik auch zu einer Auseinandersetzung mit bestehenden Theorien an, die neu gelesen und auch reformuliert werden, damit sie der jeweiligen Fragestellung gerecht werden können. Wie in Birmingham sollen auf diese Weise disziplinäre Grenzen durchbrochen werden, um neue Formen des Wissens hervorzubringen, die demokratischere und gerechtere Lebensweisen erlauben. Hierzu muss die kritische Pädagogik eine Sprache der politischen und moralischen Möglichkeiten erforschen, die den ironischen Nihilismus und Zynismus der postmodernen Sensibilität (Grossberg 1992: 224ff.) überwindet und zur politischen Partizipation führt.

Die Förderung von «multicultural literacy» ist hierbei ein besonderes Anliegen. «Central to such a pedagogy are strategies of representation, engagement, and transformation that are used to investigate the complex contours of political and social agency and how such investigations translate into providing the conditions for students and others to address in the most rigorous way possible the more urgent and disturbing issues facing them in the current historical conjuncture» (Giroux 2001: 15). Cultural Studies mit ihrem Schwerpunkt auf alltäglichen Erfahrungen und Praktiken analysieren die Bedingungen kritischer Handlungsfähigkeit, wie Grossberg (1996) zeigt, und schaffen so ein Fundament für eine praktische kulturelle Politik. So sollen vor allem Kinder und Jugendliche, die zunehmend durch die kommerzielle Konsumkultur sozialisiert werden, eine kritische Handlungsfähigkeit entwickeln, kooperative Beziehungen einüben und sich an demokratischen Werten orientieren. «Similarly, it [Cultural studies] provides an opportunity to open up spaces inside and outside the university in which teachers and students can find ways to connect knowledge to social change, while restoring, as Paul Gilroy has suggested, an ethical dimension and critical vocabulary for shaping public life as a form of practical politics» (Giroux 2001: 24f.).

Ein Schwerpunkt der an Cultural Studies orientierten «critical pedagogy» ist die «popular culture», insbesondere die Analyse von populären Filmen So deckt Giroux in dekonstruktiven und kritischen Analysen die Merkmale der Pädagogik von Hollywood auf. Er untersucht, wie Norman Denzin in seinen Analysen des Hollywood-Kinos (Denzin 1991, 2002), die mediale Politik der Repräsentation, indem er die Diskurse und Bilder von race, gender, class and sexuality analysiert. So zeigt er z. B. wie die medialen Repräsentationen von Schwarzen «a white moral panic» hervorgebrach haben (Giroux 1996). Giroux interessiert sich dafür, wie Filme und andere mediale Texte Bedeutung, Vergnügen und Identifikationen mobilisieren, 
die soziale Wirklichkeitskonstruktionen und Selbstdefinitionen beeinflussen (vgl. auch Fiske 1994). Auf diese Weise eröffnen populäre Filme aber auch einen pädagogischen Raum in einer «image saturated culture». «As the opportunities for civic education and public engagement begin to disappear, film may provide one of the few mediums left that enable conversations that connect politics, personal experiences, and public life to larger social issues» (Giroux 2002: 7).

Hier trifft sich Giroux mit der kritischen Medienpädagogik, die Douglas Kellner $(1995,2003)$ in seinen Arbeiten zur Medienkultur verfolgt. Auch er knüpft an die British Cultural Studies an, verbindet sie aber vor allem mit der Frankfurter Schule, weil er es zum einen für nötig hält, den Bereich der Produktion und die politische Ökonomie der Kultur zu berücksichtigen. Zum anderen strebt er eine Kulturkritik an, die den gegenwärtigen Moment der Geschichte theoretisch artikuliert und dabei auch seine utopischen Möglichkeiten offenlegt. «Critical theories attempt to contribute to practice and a critical cultural studies seeks to empower individuals, by giving them tools to criticize dominant cultural forms, images, narratives, and genres» (Kellner 1995: 60). Eine kritische Medienpädagogik soll die Zuschauer ermächtigen, die Botschaften, Ideologien und Werte in medialen Texten zu dechiffrieren, um der Manipulation zu entgehen und eigene Identitäten und Widerstandsformen entwickeln zu können. Darüber hinaus soll sie politisch engagierten Medienaktivismus initieren und unterstützen, um alternative Formen von Kultur und Gegenöffentlichkeiten hervorzubringen, die von entscheidender Bedeutung für eine lebendige Demokratie sind. «A democratic media politics will teach individuals how to use the new technologies, to articulate their own experiences and interests, and to promote democratic debate and diversity, allowing a full range of voices and ideas to become part of the cyberdemocracy of the future» (Kellner 1995: 337). Wie Giroux ist auch Kellner der Auffassung, dass die Medienkultur zu einer kritischen Erkenntnis der gegenwärtigen neoliberalen Verhältnisse wie der neuen globalen Ökonomie beitragen kann und durch «empowering representations» informiertere, kritischere und aktivere Formen von Subjektivität anleiten kann.

Hierzu ist vor allem die pädagogische Arbeit von Lehrern, und anderen «cultural workers» erforderlich, die ihr Wissen und ihre Kompetenz einbringen sollen, um öffentliche Räume zurückzuerobern und eine Kultur der Partizipation und der «active citizenship» zu schaffen (Giroux 1994). Durch den Erwerb von Medienliteralität in einem dialogisch und auf
Zusammenarbeit angelegten Kontext kann auch das Verständnis für andere Kulturen und Subkulturen geweckt und vertieft werden. Freilich, gehört hierzu auch eine Dekonstruktion der sozialen und politischen Konstruktion von «whiteness»: «The reality-distortion field known as 'whiteness' needs to be identified as a cultural disposition and ideology lonked to specific political, social, and historical arrangements» (McLaren 1997: 8). Es soll gezeigt werden, dass die weissen, kulturellen Praktiken kontingent und historisch produziert sind. Durch individuelles und kollektives Handeln sind sie potentiell transformierbar.

Insbesondere die neuen Medien erfordern die Entwicklung neuer Formen von «media literacy», die den interaktiven Bereichen von Computer und Multimedia angemessen sind. «Multiple literacies involve reading across varied and hybrid semiotic field and being able to critically and hermeneutically process print, graphics and representations, as well as moving images and sound» (Kellner 2002: 96). Kellner ist der Auffassung, dass eine an Cultural Studies orientierte kritische Pädagogik im neuen Millenium gerade im Bereich des Cyberspace Studenten helfen muss, ihre eigenen Räume und Interaktionsformen zu entwickeln, um das Projekt einer radikalen Demokratie zu verwirklichen.

Eine andere Richtung schlägt Peter McLaren ein. Als Reaktion auf die postfordistische Ökonomie fordert er neuerdings im Anschluss an die Arbeiten von Paulo Freire, Antonio Gramsci und Karl Marx eine «kritischrevolutionäre Pädagogik» (McLaren 2000; Allman 2001). Sie soll die Vielfältigkeit und Kreativität menschlichen Handelns in der Ära der neoliberalen Globalisierung bewahren und fördern. Um deren Marktideologie entgegen treten zu können, hält es McLaren für erforderlich, die marxistische Analyse der Gesellschaft und des Bildungswesens wieder aufzunehmen und zu vertiefen.

\section{Schluss}

Zusammenfassend lässt sich festhalten, dass die Interessen von Cultural Studies einer Kritik der Macht und einer Kunst des Eigensinns (Winter 2001) gelten, die sich z. B. in der produktiven und kreativen Auseinandersetzung mit medialen Texten und anderen kulturellen Formen in alltäglichen Kontexten entfalten können (Fiske 1993). Sie verabschieden nicht das Subjekt, sondern es geht Ihnen um eine Stärkung seiner «Agency», seiner Handlungsfähigkeit (Grossberg 1997). Wie die Arbeiten von Henry Giroux, Douglas Kellner, Peter McLaren und anderen zeigen, 
sollten Medien- und Kulturanalyse im Rahmen von Cultural Studies immer auch verknüpft sein mit einer kritischen Pädagogik, die der impliziten Pädagogik medialer Texte opponiert und eine produktive Auseinandersetzung intensivieren oder erst ermöglichen möchte. Dabei wird der Alltag als «contested terrain» bestimmt, der auf einen kollektiven Dialog hin geöffnet werden soll, damit viele unterschiedliche Stimmen sich artikulieren können, um eine demokratischere und gerechtere Gesellschaft zu schaffen.

\section{Literatur:}

Allman, Paula. (2001) Revolutionary Social Transformation: Democratic Hopes, Political Possibilities and Critical Education. Westport, Co: Greenwood Pub Group.

Apple, Michael W. (1979) Ideology and Curriculum. New York/London: Routledge \& Kegan Paul.

Apple, Michael W. (1982) Education and Power. New York/London: Routledge \& Kegan Paul.

Aronowitz, Stanley; Giroux, Henry. (1985) Education Under Siege. Massachusetts/New York: Bergin \& Garvey

Carlson, Dennis/Apple, Michael W. (Hrsg.) (1998) Power/Knowledge/ Pedagogy. The Meaning of Democratic Education in Unsettling Times. Boulder: Westview Press.

Denzin, Norman K. (1991) Images of postmodern society. Social theory and contemporary cinema. London: Sage.

Denzin, Norman K. (1994) The Art and Politics of Interpretation. In: Denzin, Norman K.; Lincoln Yvonna S. (Hrsg.) Handbook of Qualitative Research. London/ New Delhi/Thousand Oaks: Sage, S. 500-515.

Denzin, Norman K. (2002) Reading race. Hollywood and the cinema of racial violence. Thousand Oaks, Calif.: Sage.

Fiske, John. (1987) Television Culture. London/New York: Methuen.

Fiske, John. (1989) Reading the popular. Boston: Uniwin Hyman.

Fiske, John. (1993) Power Plays - Power Works. London/New York: Verso.

Fiske, John. (1994) Media matters. Everyday culture and political change. Minneapolis: Univ. of Minnesota Press.

Freire, Paulo. (1973) Pädagogik der Unterdrückten. Stuttgart: KreuzVerlag.
Frow John; Morris Meaghan. (2000) Cultural Studies. In: Denzin, Norman/ Lincoln Yvonna S. (Hrsg.) Handbook of qualitative research. Thousand Oaks, Calif.: Sage, S. 315-346.

Giroux, Henry A. (1983) Theory and Resistance in Education. Massachusetts/New York: Bergin \& Garvey.

Giroux, Henry A. (1992) Border Crossings. Cultural Workers and the politic of Education. London/New York: Routledge.

Giroux, Henry A. (1993) Living Dangerously: Multiculturalism and the Politics of Culture. New York: Peter Lang.

Giroux, Henry A. (1994) Disturbing Pleasures. Learning Popular Culture. London/New York: Routledge.

Giroux, Henry A. (1996) Fugitive Cultures. London/New York: Routledge.

Giroux, Henry A. (1999) The Mouse That Roared. Disney and the End of Innocence. Lanham/Boulder/New York/Oxford: Rowman \& Littlefield Publishers.

Giroux, Henry A. (2000) Impure Acts. The Practical Politics of Cultural Studies. London/New York: Routledge.

Giroux, Henry A. (2001) Public Spaces, Private Lives. Beyond the Culture of Cynicism. Lanham/Boulder/New York/Oxford: Rowman \& Littlefield Publishers.

Giroux, Henry A. (2002) Breaking in to the Movies. Film and the Culture of Politics. Oxford: Blackwell.

Giroux, Henry A.; Shumway, D.; Smith, P.; Sosnoski, J. (1984) «The Need for Cultural Studies: Resisting Intellectuals and Oppositional Public Spheres». Dalhousie Review 64 (2): S. 472_486.

Giroux, Henry A.; McLaren Peter. (1995) Radical pedagogy as cultural politics: beyond the discourse of critique and anti-utopianism. In: McLaren, Peter. Critical Pedagogy and Predatory Culture. Oppositional Politics in a Postmodern Era. London/New York: Routledge, S. 29-57.

Giroux, Henry A.;Lankshear Colin; McLaren, Peter; Peters, Michael. (1996) Counternarratives. Cultural Studies and Critical Pedagogies in Postmodern Spaces. London/New York: Routledge.

Giroux, Henry A./Myrsiades, Kostas(Hg.) (2001) Beyond the Corporate University. Culture and Pedagogy in the New Millenium. Lanham/ Boulder/New York/Oxford: Rowman \& Littlefield Publishers.

Grossberg, Lawrence. (1992) We Gotta Get Out of This Place. Popular Conservatism and Popular Culture. New York/London. Routledge. 
Grossberg, L.; Nelson, C.; Teichler, P. (Hrsg.). (1992) Cultural Studies. New York: Routledge.

Grossberg, Lawrence. (1996) Identity and Cultural Studies-Is That All There Is? In: DuGay, Paul; Hall, Stuart (Hrsg.). Questions of Cultural Identity. London/Thousand Oaks/New Delhi: Sage, S. 87-107.

Grossberg, Lawrence. (1997) Bringing It All Back Home. Pedagogy and Cultural Studies. In: Grossberg, Lawrence. Bringing It All Back Home. Essays on Cultural Studies. Durham/London: Duke University Press, S. 374-390.

Grossberg, Lawrence. (2000) History, imagination, and the politics of belonging: between the death and the fear of history. In: Grossberg, Lawrence; Gilroy, Paul; McRobbie, Angela (Hrsg.). Without Guarantees. In honour of Stuart Hall. New York/London: Verso Books, S. $148-164$.

Hall, Stuart. (1981) Notes on deconstructing the popular. In: Samuel, R. (Hrsg.). People's history and social theory. London: Routledge \& Kegan, S.227-240.

Hay, James; Grossberg, Lawrence; Wartella, Ellen (Hrsg.). (1996) The Audience and Its Landscape. Boulder: Westview Press.

Kellner, Douglas. (1995) Media Culture. Cultural Studies, Identity and politics between the Modern and the Postmodern. London/New York: Routledge.

Kellner, Douglas. (2001) Critical Pedagogy, Cultural Studies, and Radical Democracy at the Turn of the Millenium: Reflections on the Work of Henry Giroux. Cultural Studies-Critical Methodologies, Vol. 1, number 2, May 2001, S. 220-239.

Kellner, Douglas. (2002) New Media and New Literacies. Reconstructing Education for the New Millenium. In: Lievrouw, Leah H.; Livingstone, Sonia (Hrsg.). Handbook of New Media. Social Shaping and Consequences of ICTs. London/Thousand Oaks/New Delhi: Sage, S. 90-104.

Kellner Douglas. (2003) Media spectacle. London: Routledge.

McLaren, Peter. (1986) Schooling as a Ritual Performance. Towards a Political Economy of Educational Symbols and Gestures. London/New York: Routledge.

McLaren, Peter. (1995) Critical Pedagogy and Predatory Culture. Oppositional Politics in a Postmodern Era. London/New York: Routledge.
McLaren, Peter. (1997) Revolutionary Multiculturalism. Pedagogies of Dissent for the New Millenium. Boulder: Westview Press.

McLaren, Peter. (2000) Che Guevara, Paulo Freire and the Pedagogy of Revolution. Lanham/Boulder/New York/Oxford: Rowman \& Littlefield Publishers.

Steele, Tom. (1997) The Emergence of Cultural Studies 1945-65. Cultural Politics, Adult Education and the English Question. London: Lawrence \& Wishart.

Williams, Raymond. (1989) The Future of Cultural Studies. In: Raymond, Williams. The Politics of Modernism: Against the new Conformists. London: Verso, S.151-162.

Willis, Paul. (1977) Learning to Labour: How Working Class Kids Get Working Class Jobs. Farnborough: Saxon House.

Winter, Rainer. (2001) Die Kunst des Eigensinns. Cultural Studies als Kritik der Macht. Weilerswist: Velbrück Wissenschaft. 